\title{
Possible Mechanism of the Sex Differentiation and Its Artificial Regulation
}

\author{
A.I.Ibraimov \\ Laboratory of Human Genetics, Kyrgyz National Center of Cardiology and Internal Medicine, \\ Togolok Moldo Street 3, KG-720 040 Bishkek, Kyrgyzstan \\ Telephone: + (996 312) 62-27-61, Fax+(996 312) 66-03-87; \\ E-mail: ibraimov_1@msn.com
}

KEYWORDS Sex differentiation; cell thermoregulation; constitutive heterochromatin; sex regulation; X-chromosome inactivation

\begin{abstract}
Seemingly, the sex differentiation (SD) in animals and human is determined by the amount of constitutive heterochromatin region (HR) in the chromosomes of the undifferentiated embryonic gonads (UEG) via cell thermoregulation. It is assumed the medulla and cortex tissue cells in the UEG differ in vulnerability to the increase of the intracellular temperature. If the amount of the HR is enough for efficient elimination of heat difference between the nucleus and cytoplasm in rapidly growing UEG cells the medulla tissue survives. Otherwise it doomed to degeneration and a cortex tissue will remain in the UEG. For artificial regulation of the SD it is proposed to remove a layer of cortex or medulla in the UEG depending on the objective and task of the research.
\end{abstract}

\section{INTRODUCTION}

"The function of heterochromatin must be involved in the differences between somatic and germinal cells"

- Richard Goldschmidt

In many animals, including us, the genetic sex is determined at fertilization by sex chromosomes carried by the father's sperm, $\mathrm{X}$ in the case of female and $\mathrm{Y}$ in the case of male. In birds, moths, and butterflies, males are $\mathrm{XX}(\mathrm{ZZ})$ and females are $\mathrm{XY}(\mathrm{XW})$.

However at the early stages of embryonic development a pair of undifferentiated embryonic gonads (UEG) and both rudimentary female and male reproductive system develops in the embryo. As result of this all embryos are potentially bisexual.

For the time being the mechanisms of the sex differentiation (SD) are not known. At present the balance hypotheses, worked out by Bridges (1939) and Goldschmidt (1955) are generally accepted. According to these hypotheses, the interaction of genes, located in the sex chromosomes and autosomes, underlie the SD. Thus, it is considered that sex is a polygenic features.

However, almost nothing is known about concrete mechanisms and types of gene interaction at the SD. The problem also becomes complicated especially as: a) the number, localization, products, and types of these gene interactions are not determined; $b$ ) the role of the sex chromosomes in the embryo SD remains not completely clear; c) there are no ideas as regards the possible role of a great amount of constitutive heterochromatin region (cHR) of the Y chromosome in the SD.

\section{HETEROCHROMATINANDONTOGENESIS}

Very few special researches on the peculiarities of organization and behavior of the heterochromatin regions (HRs) in process of the individual development and differentiation of the cell systems have been carried out. The available data are mainly restricted by separate stages of ontogenesis with some organisms.

Boveri (1888) discovered the heterochromatin diminution in the mitotically division cells in Ascaris. Later the heterochromatin diminution was found in cyclops, infusoria, Stylonychia, Miaster, Sciaria, Phynchosciria and Ceccidonidae.

In mitosis of the early embryogenesis the amplification of highly repetitive DNAs sequences creating large blocks of the HRs occur. With all objects, well studied at the early stages of embryogenesis - cyclops, loach, salmon, trout, a number of amphibians, mice - the interphase nuclei cells of the very first divisions of the egg cleavage possess remarkable peculiarities. At stage 2 to 4 blastomeres the heterochromatin is not revealed. All parts of the chromosomes in these nuclei are despiralyzed, and the nucleolus 
is missing. At the metaphase stage the chromosomes represent thin filament structures. The gradual nucleus structuring and develop-ment of nucleolus accompany the successive cleavage of the blastomeres, and the chromo-somes in the metaphase acquire a morphological structure that is peculiar to this species (for review see Prokofyeva-Belgovskaya 1986).

In the ontogenesis of the somatic cells the chromosomes undergo progressive heterochromatinization. Burkholder and Comings (1972) in comparing the $\mathrm{G}+$ bands of the chromosomes of the later embryonic fibroblasts and chromosomes of blastocyst have found a more intensive and larger centromere HRs in the embryonic fibroblasts and at the same time a complete correspondence of the G-bands pattern by the chromosomes length in both types of the cells.

Lyon (1961) proposed the single-active X chromosome hypothesis to explain the observation that in the mouse, females heterozygous for X-linked fur color genes are patchy mosaics of two colors. To quote Lyon: “... (1) that the heteropicnotic X-chromosome can be either paternal or maternal in origin in different cells of the same animal; (2) that it is genetically inactivated". According to the Lyon this mechanism provides dosage compensation for X-linked genes because each cell, male or female, has only one $\mathrm{X}$-chromosome that is transcribed. This generally accepted thesis connects the main reason of inactivation of one $\mathrm{X}$ chromosome in the normal female with possible undesirable genes' effects in case they are in double amount in the mammal's genome.

As we conceive, the hypothesis of gene dosage compensation may turn out to be not the only reason for "lyonization". It is possible that in this phenomenon the heat conductivity effect of the condensed chromatin (CC) in the cell thermoregulation also plays quite an important role (for details see Ibraimov 2003, 2004; Ibraimov and Tabaldiev 2007). In short, the essence of our objections is based on the following facts:

1) the inactivation of one $X$-chromosome in the normal females occurs only with the mammals, which are able to support a relatively constant core temperature in the body;

2) the inactivation of $X$ chromosome takes place early in embryonic development at an estimated 1000 - to 2000- cell stage of the blastocyst or possibly even earlier (for review see Lyon, 1974), i.e. when the size of the cells are considerably smaller than that of the fertilized egg, and they become more or less closer to the size of the somatic cells. As is known the cell size is restricted by the surface-to-volume ratio, as a cell metabolizes, it must exchange materials and heat with its environment. In other words, with the formation of a multicellular embryo the problems arise, which are connected with the intracellular thermoregulation, and we believe that for their solution the $\mathrm{CC}$ is of great importance (Ibraimov 2003, 2004). As in thekaryotype of the female mammals there is lack of the sex chromosome with a large block of cHR, for example as Y chromosome with males, then it is possible that in the interest of the cell thermoregulation a considerable part of one of the two X-chromosomes with the female embryo is undergoing to heterochromatinization;

3) it is known that at the level of population there is no significant differences between sexes by the amount of C- and Q-HRs on the autosomes (Ibraimov and Mirrakhimov 1985). However, it is established that the amount of Q-HRs on the Y chromosome influences to the mean number of Q-HRs per individual $(m)$, for example in males with large blocks of Qheterochromatin on the $\mathrm{Y}$ chromosome, the value of $m$ on their autosomes is lower and vice versa (Ibraimov et al. 2000). These observations indirectly justify the possibility of existence of some mechanisms of compensation of a hetechromatin "dose" in the genome between sexes. If such assumption is correct than the heterochromatinization of one of the two Xchromosomes in cells of the mammals could have a rational explanation within the framework of the hypothesis on participation of the $\mathrm{CC}$ in the cell thermoregulation. In this case the female genome via heterochromatinization of a greater part of the body of one of the two X-chromosomes compensates the lack of Y chromosome with a large block of cHR in its karyotype. And a certain trend to the increase of the value of $m$ in females (Ibraimov 1983) justify that the heterochromatinized X-chromosome (facultative heterochromatin) possibly not completely replaces the heat conductive effect of the Y chromosome cHR in the interphase nucleus. This is proved by the physiological data on a relatively low heat conductivity of the female body in 
comparison with the males' one (Ibraimov and Tabaldiev 2007);

4) in normal females, 30 to 50 percent buccal cells show a Barr body, but the count from different laboratories very greatly. In cultural fibroblasts, the incidence of cells with Barr bodies is more than 90 percent when most of the cells are in the $\mathrm{G} 2$ phase. In polymorphonuclear white blood cells the inactive X chromosome appears as a drumstick-shaped extrusion with a frequency of 1 to 10 percent. Y-body morphology may vary with DNA synthesis activity, being more dispersed in cells with active DNA synthesis. From this it is possible to assume that the X-and Y-bodies do not appear in the G1 phase when the cell for the exact implementation of its specific function is in need of preservation of the intracellular temperature homeostasis most of all. Apparently, in the G1 phase of the cell cycle both the constitutive and facultative heterochromatin is in the composition of the of the peripheral layer of the $\mathrm{CC}$ in the most compacted state in order to efficiently equalize the temperature difference between the cytoplasm and nucleus (Ibraimov 2003, 2004). In this sense the X-and Y-bodies do not participate in the cell thermoregulation, as in this form they occupy only a small part of the surface of the nuclear envelope in spite of their dense states, especially since they are formed after the chromosome replication;

5) if compensation (double) dose of genes is an inevitable phenomenon for normal functioning of the mammals' genome, then why the inactivation of genes on the homologous autosomes do not happen? The inactivation of autosomes was not found even in cases of trisomies. Yunis (1977) pointed out that the only three autosomes (13, 18 and 21) for which trisomy are viable to any appreciable extent. A similar nonrandomness has been shown for spontaneous trisomic abortions. Yunis concluded that these chromosomes contained more heterochromatin, therefore, their trisomies were better tolerated than trisomies fore more generic autosomes.

6) "...the mammalian X chromosome is not specialized for sex determination. Although a rather large number of X-linked genes are known in mammals, a vast majority of them have nothing whatsoever to do with the process of sex determination and sexual development. On the contrary, many of the genes clearly involved in sexual development reside in autosomes"... .'Even the genes for hypothalamic releasing factors of gonadotropins are apparently on autosomes" (Ohno 1983).

Apparently, the hypothesis of the gene dosage compensation does not cope with the task of explanation of the above-mentioned facts completely. It is possible that the main reason of the"lyonization" is not the gene dosage compensation, but the compensation of the "dose" of the cHR, missing in the genome of the mammals' females for efficient leveling of the temperature difference between the nucleus and cytoplasm that is very important for keeping the temperature homeostasis with the mammals (Ibraimov 2003, 2004; Ibraimov and Tabaldiev 2007). In other words the inactivation of the Xchromosome in the mammals' females occurs, mainly, in the interests of thermoregulation.

In order to clarify the essence of our SD hypotheses, it is necessary to remind, in brief, some generally known facts of the sexual development. The sexual development in the mammals is a process consisting of at least three stages: the $1^{\text {st }}$ stage is the chromosome determination of sex (XX or $\mathrm{xy})$; the $2^{\text {nd }}$ stage is $\mathrm{SD}$ (the development of testicles or ovaries); the $3^{\text {st }}$ stage is the development of the secondary sexual characteristics. In case the testicles are formed, they excrete the testosterone hormone, circulating over embryo, and causing the development of the somatic cells by the male type. On the contrary, the lack of testosterone results in invariable development of the somatic cells by the female type. Normally, the direction of the sexual development is determined by availability of the Y chromosome; the male gonads are developing if there is one $\mathrm{Y}$ chromosome irrespective of the $\mathrm{X}$ chromosome number. It is assumed that the formation of testicles is a result of direct effect of the genes located in the $\mathrm{Y}$ chromosome. By another concept the sexual development may also depend on the $\mathrm{H}-\mathrm{Y}$ antigens, though lately its role calls in question.

The UEG turn out to be of dual nature, or to be more exact they are indifferent concerning sex. They consist of the outer layer of tissue (cortex) from which the female tissue develops, and the inner layer, called medulla, from which the male tissue develops. In course of the $2^{\text {nd }}$ stage of the sexual development, the progress of one of the 
germs and suppression of the other one takes place. In the male sex the medullary tissue, which suppresses the activity of the cortex layer, develops quicker; as a result the gonads turn into the testicles.

We assume that basically the SD is a "physical process", and at this stage of the sexual development the role of genes (a chemical process) is insignificant. The genes effects mainly determine the development of secondary sexual characteristics.

Our hypothesis is guided by a concept of the cell thermoregulation (CT) and the role of the cHR in the composition of the condensed chromatin (CC) (Ibraimov, 2003) in this process. The essence of the CT is in the following: CC, being the densest domains in a cell, apparently conducts heat between the cytoplasm and nucleus when there is a difference in temperature between them. The assumed heat conductivity effect of CC is stipulated by its principal features: condensed state during the interphase, association with the lamina and the inner nuclear membrane, replication at the end of the $S$ period of a cell cycle, formation of the chromocenter, genetic inertness, and wide variability in the quantitative contents both within and between species (Ibraimov 2003). The reality of the CT existence is shown at the organism level. In particular, it turned out that the individuals in the population differ from one another by the heat conductivity of their bodies (BHC). At that the BHC value depends on amount of the Q-HRs in their genomes (Ibraimov and Tabaldiev 2007).

As we conceive, the cHR plays an important role in the SD. Let's try to illustrate this assumption on the example of a human being. Until now the hypothetical genes responsible for the development of the male sex in the Y chromosome have not been revealed. The point is that the Y chromosome is largely a dummy (Ohno 1983). Most likely from our point of view, the cHR of the Y chromosome are responsible for the development of UEG towards formation of the testicles, and not some genes or the H-Y-antigen.

At first we give some initial prerequisites. (1). Whatever the nature of the factors determining the male sex may be, they are necessary in order that the UEG develop as the testicles, which is the first step in a male development. For lack of the processes causing the testicles development, the UEG develops invariably as an ovary. (2). SD at the level of gonads turned out to be a threshold phenomenon; to transform the germ cells of the gonads into the testicles some minimum "dose" of the factor switching over the direction of the sexual development is needed. (3). The sex "genotype" manifests its direct impact only at this stage of the UEG development transferring the further control over the corresponding development of the secondary sexual characteristics to different hormones. (4). The $\mathrm{X}$ chromosome of the mammals remains evolutionary stable; the genes located in it are homologous in all studied species of mammals (Ohno 1983). (5). It is known that the amount of cHR in males at the level of population is on average two times more than in females (Paris conference 1971, 1975; Ibraimov and Mirrakhimov 1985). (6). Most of the genes responsible for the development of the secondary sexual characteristics are concentrated not on the sex chromosomes, but on the autosomes (Ohno 1983).

Now let's try to ground our assumption. (1). The heat conductive effect of the CC especially strongly increases in conditions of mulicellularity (Ibraimov 2004); (2). By the $3^{\text {rd }}$ week of the embryo development in human, the HRs is completely formed [Prokofyeva-Belgovskaya 1988], and they are able to exert their heat conductive effects in the cells. (3). In number mammals, including man, it has been shown that at equivalent gestational ages, males are developmentally more advanced than females. Medulla, being located in the very middle of the UEG closed to aorta and surrounded with mesentery probably experiences the greatest problems with removal of the excessive heat in comparison with cortex. Obviously, the cortex having a relative advantage in supporting the intracellular temperature homeostasis than the medulla, other things being equal, has more chances to preserve and further develop into the female tissue. (4). Physical agents may determine sex as temperature. For example as early as in the first decades of the XX century in the tests with the descendents of the triploid females of Drosophila it was shown that at high temperature the flies with the female characteristics are being developed, and at a lower temperature - with the male sexual characteristics. Conover and Kynard (1981) have found that in the fish Menidia menidia (Atlantic Silverside fish) sex determination is under genetic and temperature control during a critical phase of larval development. The sex ratios of the progenies were highly skewed by this treatment. The eel (Anguilla) has no chromosomes that can be recognized as sex 
chromosomes and it shows the same skewed sex ratios as Menidia (Wiberg 1983). There are many such examples in animals (Lima-de-Faria 1983).

It is possible that the medulla of the UEG is more vulnerable to the temperature increase than the cortex tissue. The following data testify to this: a) the clinical consequences of cryptorchidism in boys; b) location of scrotum outside the body in the mammals. In other words, the direction of development of the UEG towards the male or female side is not something strictly fixed, it will depend on the environment in which it is being implemented. In our opinion, most likely this is the temperature influence on the UEG tissues, and seemingly on which it depends, whether the medulla tissue will remain or not. If the medulla tissue is not provided with the timely and efficient removal of excessive heat, it will be doomed to degeneration having making for the cell from the cortex. Just in this meaning we understand the role of cHR in SD, as efficient means of medullar tissue protection against "heat death". As there is a wide intrapopulation polymorphism by the amount of cHR in the $\mathrm{Y}$ chromosome (Paris Conference, 1971, 1975), no wonder that even insignificant deviation in the operation of the system ensuring the SD at the gonad level so often causes an incomplete development of the male phenotype in the organism with the male genotype (Vogel and Motulski 1986).

\section{DISCUSSION}

According to Lyon (1992) " "... the X-chromosome is clearly involved at some point in the sexdetermination pathway". The point that I am trying to convey is that: a) the SD is one of the most important examples of how the physical state of the DNA molecular (in this case the level of compacting of the peripheral layer of $\mathrm{CC}$ around the cell nucleus) influences to the cell differentiation; b) X-inactivation is not involved in the SD; c) $\mathrm{x}$ - chromosome is not being inactivated, but it is heterochromatinized in order to compensate the lacking in the female karyotype the largest block of the $\mathrm{cHR}$ in the interest of the CT. Thus it would be more correct to speak about compensation of the heterochromatin dosage, and not about the dosage (double) of genes.

The reasons why the cortex tissue in the UEG is not exposed to the heat degeneration as the medullar tissue because of the problems connected with the $\mathrm{CT}$ in addition to the above mentioned, are also explained by the fact that: a) it has been shown that at equivalent gestational ages, males are developmentally more advanced than females, even before the gonads form. So, as for example a detailed analysis of XX v. XY developmental differences in mice has shown that XY fetuses are indeed larger than XX fetuses prior to gonadal SD (Burgoyne et al. 1995). The human testicles are formed earlier $\left(6^{\text {th }}-12^{\text {th }}\right.$ week $)$ than the ovaries $\left(14^{\text {th }}-16^{\text {th }}\right.$ week $)$; b) by the time of the ovaries formation, the problems with heat conductivity out from the deep parts of the organs and embryo tissues become considerably less complicated, as by this time the circulation system of fetus begins to function, i.e. a distant transfer of heat energy in a body (Ibraimov and Tabaldiev 2007), that eliminates the danger of the cell heat degeneration including the cortex tissues; c) with mammals and human the development towards the male sex is determined by availability of a testicle, to be more precise by a hormone secreted by it - testosterone, and the development towards the female sex is not induced, i.e. it is not the consequence of availability of the embryo ovary. It rather should be considered as the consequence of the testicle absence (Ohno et al. 1971); d) random Xinactivation of either the maternally- or paternally - inherited X chromosome most likely justifies to the participation of the facultative heterochromatin in the $\mathrm{CT}$, and not to the dosage compensation of genes; and last c) homeotherms keep the constant temperature in a body not so much they possess a 4-chamber heart and lung respiration (as a result the tissues obtain more oxygen) for acceleration of heat production, as their ability to efficiently remove the excessive heat from the organism. This is promoted by high conductivity of their bodies which are provided, in addition with the $\mathrm{cHR}$, with the availability of $\mathrm{G}+$ and $\mathrm{Q}+$ bands in the chromosomes which intensify the compactization of the peripheral CC layer in the interphase nuclei, thus accelerating the liquidation of the appeared temperature difference between the nucleus and cytoplasm in the cell (Ibraimov 2003, 2004). So, the cause of the differential survivability of the medulla and cortex cells is the $\mathrm{CT}$ (preservation of the intracellular temperature homeostasis), which is determined by the amount of the HRs.

Within the framework of our hypothesis the results of the classical experiments of Bridges (1939) may be interpreted. In any manual on 
genetics a table, where for each sex type of $D$. melanogaster the number of the $\mathrm{X}$ chromosomes, number of autosomes - so called sex index is given. Also there is a figure illustrating a chromosome set of various sex types, which occur in the offspring of the triploid fly's females. As we argue the role of the genes effect in the $\mathrm{SD}$, we will not analyze the table data, and will restrict ourselves only to the karyotypes of different sex types. So, in Fig. 1 on top, the flies karyotypes according to Bridges within the frame of his hypothesis of the gene balance is shown, and at the bottom of the Figure there is our interpretation of the same karyotypes taking into consideration only the amount of the cHR in all the D. melanogaster chromosomes without

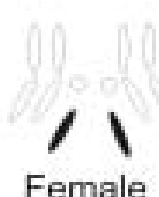

Female

A.

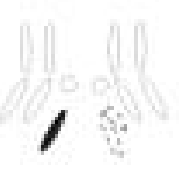

Male

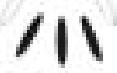

Superfemale

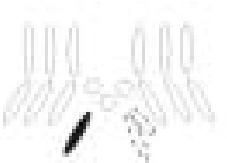

Supermale
11

Triploid
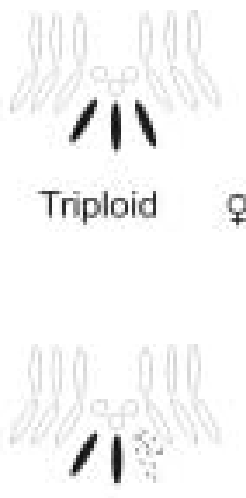

Triploid
B.
Female
Superfemale

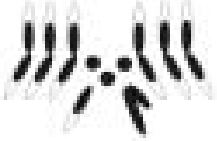
Supermale
Triploid

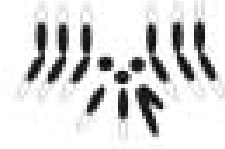
Triploid

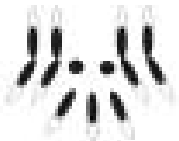

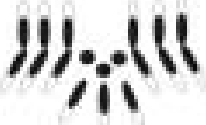

Fig. 1. A chromosomal complement of the different sexual types in offspring of the triploid females of $D$. melanogaster. A. It shows the importance of balance of $\mathrm{X}$ chromosomes (black) and autosomes - II, III, and IV (white) in sex differentiation. The $Y$ chromosome, which is not influencing on sex differentiation is represented by a dotted line (by Bridges). B. It shows the relative sizes of heterochromatin (black) in all without exception chromosomes to illustrate the value of amount of constitutive heterochromatin in sex differentiation. 
exception. There are several necessary remarks on Figure 1: a) as well as in Bridges's work, the outlines of the $\mathrm{Y}$ chromosome are marked by dotted lines. It is always done in order to specially underline its insignificance in the SD; b) the relative parts of the area, occupied by the cHR by the length of each $D$. melanogaster chromosome are taken from the work of ProkofyevaBelgovskaya (1986); c) for the analysis convenience, the total amount of the cHR in the karyotype of a normal diploid fly was taken to be 100 , and this total was distributed for the individual chromosomes as follows: - shares of the cHR for chromosomes 3,2, 4, X and Y amounted to 10, 10, 5, 25 and 50 conventional units, respectively. In Figure 1 the areas, occupied by the cHR on the corresponding chromosomes, are shared.

If in such calculation the total number of the cHR with a normal diploid male of the $D$. melanogaster is 125 conventional units (c.u.), then in a female it is 100 c.u., i.e. by $20 \%$ less then in a male. Approximately the same ratio is observed in so called super-females and super-males (125 and 150 c.u.), and in triploid females and males it is 150 and 175 c.u., respectively. Thus, in all cases the females have the cHR approximately by $20 \%$ less than the males, irrespective of the ploidy of the autosomes and sex chromosomes number in the karyotype. These results seem to prove that the $\mathrm{SD}$ in the animals is most likely determined by the total amount of the cHR in the embryo genome, but not by the gene balance, which is located in the $\mathrm{X}$ chromosome and autosomes.

It could be possible to test our hypothesis experimentally. At UEG with the karyotype XX to remove its cortical layer preserving the medulla tissue. If our hypothesis is true then a male with a female genotype (XX) will be developed, which at usual crossing results only in females. Such experiments could give an answer to two interrelated question: 1) what does the SD depend on, either on the gene balance or on the "dose" of the cHR?; 2) why does at genotype XX the medulla tissue preliminarily degenerate?, either from the "heat death" because of a small dose of the $\mathrm{cHR}$ or from the impact of the gene products, produced by the cortex cells, on the medulla tissue? This principle is also acceptable for birds, which females are heterogamete, for example in order to have only females in the offspring. For this, in the embryo with genotype $\mathrm{ZZ}$ it is necessary to remove the medulla tissue and preserve the cortex to have a hen in the offspring, which produces only the female gamete.

\section{ACKNOWLEDGEMENTS}

I apologize to those authors, whose work were not cited, or were cited only through reviews. It was only because of the space limitations. I am grateful to S. Tabaldiev for excellent technical assistance.

\section{REFERENCES}

Boveri T 1888. In Die Befrunchtung und Teilung des Eies von Ascaris megalocephala. Zellen-Studien. Jena: G. Fisher. H. 2, S., pp.1-189.

Bridges CB 1925. Sex in relation to chromosomes and genes. Amer Nat, 59: 127-137.

Burgoyne PS, Thornhill AR, Kalmus Boudreau S, Darling SM, Bishop CE, Evans EP 1995. The genetic basis of XX-XY differences present before sex differentiation in the mouse. Philos Trans Roy Soc Lond Biol, 350: 253-261.

Burkholder GD, Comings DE 1972 Do the Giemsabanding patterns of chromosomes change during embryonic development? Exp Cell Res, 75: 268271

Conover DO, Kynard BE 1981. Environmental sex determination: interaction of temperature and genotype in fish. Science, 213: 577-579.

Goldschmidt R 1955. Theoretical Genetics. Berkeley and Los Angeles: University of California Press.

Ibraimov AI 1983. Human chromosomal polymorphism. VII. The distribution of chromosomal Q-polymorphic bands in different human populations. Hum Genet, 63: 384-391.

Ibraimov AI 2003. Condensed chromatin and cell thermoregulation. Complexus, 1: 164-170.

Ibraimov AI 2004. The origin of condensed chromatin, cell thermoregulation and multicellularity. Complexus, 2: 23-34.

Ibraimov AI, Mirrakhimov MM 1985. Q band polymorphism in the autosomes and $\mathrm{Y}$ chromosome in human populations. In: A.A. Sandberg (Ed.): Progress and Topics in Cytogenetics. The $Y$ chromosome. Part A. Basic characteristics of the $Y$ chromosome. New York: Liss. pp. 213-287.

Ibraimov AI, Tabaldiev SK 2007. Condensed chromatin, cell thermoregulation and human body heat conductivity. J Hum Ecol, 21(1): 1-22.

Ibraimov ÀI, Karagulova GO, Kim EY 2000. The relationship between the $\mathrm{Y}$ chromosome size and the amount of autosomal Q-heterochromatin in human populations. Cytobios, 102: $35-53$

Lima-de-Faria A 1983. Molecular Evolution and Organization of the Chromosome. Amsterdam: Elsevier.

Lyon MF 1961. Gene action in the X-chromosome of the mouse (Mus musculus L.). Nature, 190: 372373

Lyon MF 1974. Mechanisms and evolutionary origins of variable $\mathrm{X}$ - chromosome activity in mammals. Proc $R$ Soc Lond B, 187: 243-268. 
Lyon MF 1992. Some milestones in the history of Xchromosome inactivation. Annu Rev Genet, 26: 17-28.

Ohno S 1983. Phylogeny of the X chromosome of man. In: A.A. Sandberg (Ed.): Cytogenetics of the mammalian $X$ chromosome, Part A. Basic Mechanisms of $X$ chromosome behavior. New York: Liss. pp.1-19.

Ohno S, Tettenbroun U, Dofuku R 1971. Molecular biology of sex differentiation. Hereditas, 69: 107124.

Paris Conference, 1971, Supplement, 1975. Standartiza- tion in human cytogenetics. Birth Defects, XI: 1-84. Prokofyeva-Belgovskaya AA 1986. Heterochromatic Regions of Chromosomes (in Russian). Moscow: Nauka.

Vogel F, Motulsky AG 1986. Human Genetics. Problems and Approaches. Berlin, Heidelberg, New-York, Tokyo. Springer-Verlarg.

Wiberg UH 1983 Sex determination in the European eel (Anguilla anguilla, L.). Cytogenet Cell Genet, 36: 558-598.

Yunis JJ 1977. New Chromosomal Syndromes. New York: Academic Press. 